evaluations of lessened seizure severity $(\mathrm{p}=0.019)$. Adverse effects occurring more frequently among TPM treated than placebo-treated children included emotional lability ( $12 \%$ vs $4 \%$ ), fatigue ( $15 \%$ vs $7 \%)$, impaired attention (12\% vs $2 \%)$, and impaired memory ( $7 \%$ vs $0 \%$ ). Side-effects were not sufficiently severe to require discontinuation of therapy. TPM was considered safe and effective as an adjunctive treatment of partial-onset seizures in children. (Elterman RD, Glauser TA, Wyllie E et al. A double-blind, randomized trial of topiramate as adjunctive therapy for partial-onset seizures in children. Neurology April 1999;52:13381344). (Reprints: Dr Roy Elterman, Dallas Pediatric Neurology Associates, $12801 \mathrm{~N}$ Central Expressway, Plaza 3, Suite 580, Dallas, TX 75230).

COMMENT. In addition to trials in children with partial seizures, topiramate ( $6 \mathrm{mg} / \mathrm{kg} /$ day) as adjunctive therapy has been tested and proven effective and well-tolerated in children and adults with primary generalized tonic-clonic seizures (Biton V, Montouris GD, Ritter F et al. A randomized, placebo-controlled study of topiramate in primary generalized tonic-clonic seizures. Neurology April 1999;52:1330-1337).

\title{
VIGABATRIN vs CARBAMAZEPINE IN PARTIAL SEIZURES
}

The efficacy and tolerability of vigabatrin (VGB) and carbamazepine (CBZ) monotherapy were compared in an open long-term study in newly diagnosed partial seizures in children treated at the Infantile Neuropsychiatric Division of the Regional Pediatric Hospital, Ancona, Italy. VGB, 50-60 mg/kg/day. in 38 patients or CBZ, $15-20 \mathrm{mg} / \mathrm{kg} /$ day, in 32 patients were split into twice-a-day doses given for a 2-year follow-up period. Clinical efficacy of VGB was similar to that of CBZ ( $76 \%$ vs $78 \%$ benefited, respectively). Relapses occurred in $24 \%$ of the VGB group and $22 \%$ of the CBZ-treated patients.

VGB adverse effects included abnormal weight gain of $10-20 \%$ in $10(26 \%)$ patients within the first 3 months of the study, irritability/excitability and antisocial behavior in $6(16 \%)$, and asymptomatic visual field constriction in an unspecified number. The most significant and troublesome CBZ adverse effect was a generalized urticarial rash within the first 10 days of therapy in $6(19 \%)$ patients, associated with high fever and severe leukopenia in one. Excessive sedation occurred in 6 (19\%). Effects on cognitive function were not addressed. (Zamponi N, Cardinali C. Open comparative long-term study of vigabatrin vs carbamazepine in newly diagnosed partial seizures in children. Arch Neurol May 1999;56:605-607). (Reprints: Nelia Zamponi MD, Department of Pediatric Neurology, "G Salesi" Children's Hospital, Via Corridoni, 11, 60 123, Ancona, Italy).

COMMENT. The clinical efficacy of vigabatrin and carbamazepine monotherapy was comparable in an open long-term trial in children with newly diagnosed partial seizures. The high incidence of skin rash within 10 days of initiating CBZ therapy is a concern, and the role of VGB as an alternative treatment of childhood partial epilepsies should be studied further. The value of VGB as therapy for infantile spasms has been established and reported previously.

VGB response rate and tolerability were superior to other new antiepileptic drugs (gabapentin, lamotrigine, tiagabine) in a meta-analysis comparison of key clinical trials (Cramer JA, Fisher R, Ben-Menachem E, French J, Mattson RH. New antiepileptic drugs: comparison of key clinical trials. Epilepsia May 1999;40:590$600)$. Drawbacks to the use of these data included significant differences among trials in reports of placebo response; lower than average response $(6 \%)$ in tiagabine trials, and higher than average response $(14 \%)$ in topiramate trials. 
Also, drug response rates and incidence of adverse effects differed between study populations, despite use of identical protocols and patient selection.

The management of refractory seizures and their effects on the quality of life of the patient are reviewed (Devinsky O. Patients with refractory seizures. $\underline{\mathrm{N}}$ Engl _ Med May 20 1999;340:1565-1570). The optimal use of antiepileptic drugs, first-line and second-line choices, and their effects on other drug serum levels are tabulated. Vagus-nerve stimulation, recently approved as an adjunctive therapy for refractory seizures in patients older than 12 years, is discussed.

\section{SEIZURE DISORDERS}

\section{VAGUS NERVE STIMULATION FOR REFRACTORY EPILEPSY}

The efficacy of vagus nerve stimulation (VNS) was evaluated in 24 patients, aged 4 to 40 years (median 18 years), at the New York Presbyterian HospitalCornell Medical Center, NY; Mercy Children's Hospital, Kansas City; and University of California at San Diego. Seizure rates during a 1-month baseline were compared to those with 3 months of VNS. Improvements occurred in 22 (88\%); 16 had $>30 \%$ reduction, and 11 had $>50 \%$ reduction in seizure rate. The median seizure rate reduction was $46 \%$. Idiopathic epilepsy patients improved more than those with symptomatic epilepsy (-60\% cf $-40 \%)$. Generalized tonic seizures responded better than generalized tonic-clonic seizures $(-70 \%$ of $-33 \%)$. Patients with higher baseline seizure rates responded better. Age at onset of epilepsy (median 2, range 0-14 years) was also a predictor of response; seizures developing in later childhood were more responsive.

Adverse events included cough (6 patients), abdominal pain (2), and anorexia, hiccups, dysphagia, emesis, and fatigue (1 each). All were considered mild except one moderate cough and one with anorexia. The median heart rate was slowed compared to baseline. (Labar D, Murphy J, Tecoma E, E04 VNS Study Group. Vagus nerve stimulation for medication-resistant generalized epilepsy. Neurology April 1999;52:1510-1512). (Reprints: Dr Douglas Labar, Comprehensive Epilepsy Center, New York Hospital-Cornell Medical Center, K-619, 525 E 68th Street, New York, NY 10021).

COMMENT. Vagus nerve stimulation may be indicated in older children and adolescents with drug-refractory epilepsies, especially generalized tonic seizures. This pacemaker device, connected by two stimulating electrodes to the left vagus nerve, appears to be safe and generally well tolerated. Transient hoarseness is the most common adverse effect, but cough and anorexia may also occur. Further studies are needed to define the types of seizures responsive to VNS in children.

\section{OUTCOME OF EPILEPSY SURGERY IN EARLY CHILDHOOD}

The medical records of 23 children, ages 0-3 years, who were treated surgically for epilepsy between 1991 and 1996 were analysed at the Hospital for Sick Children, Toronto, Canada. The mean age at onset of seizures was 4.7 months, and the mean age at time of surgery was 15.3 months. Partial seizures were diagnosed at onset in 20, infantile spasms in 2, and generalized tonic-clonic seizures in one. Focal cortical resection was performed in 21 and hemispherectomy in 11. Pathological findings included focal cortical dysplasia ( 8 patients), Sturge-Weber syndrome (5), hemimegalencephaly (3), low-grade glioma (3), and tuberous sclerosis (1). Seizure outcome was class I in 12, class II in 3 , class III in 6, and class IV in 2 (Engel's criteria). Outcomes for Sturge-Weber and low-grade glioma patients were better than those with neuronal migration disorders (NMD). Patients with NMD who did poorly had normal MRI/CT findings 